\title{
Psycholinguistic and Psychometric Measurements of the Turkish Pediatric Epilepsy Medication Self- management Questionnaire
}

\author{
(D) Şerife Tutar¹, (1) Ayşegül İşler Dalgıç2 \\ 1Süleyman Demirel University Faculty of Health Sciences, Department of Pediatric Nursing, Isparta, Turkey \\ ${ }^{2}$ Akdeniz University Faculty of Nursing, Department of Pediatric Nursing, Antalya, Turkey
}

\begin{abstract}
Aim: The aim of this study was to examine the psychometric and psycholinguistic characteristics of "The Pediatric Epilepsy Medication Selfmanagement Questionnaire" which was adapted into Turkish.

Materials and Methods: The sample of this methodological study consisted of 540 parents whose children (between the ages of 2-17 years) were followed up at Akdeniz University Hospital Pediatric Neurology Polyclinic between April 2015 and June 2016 with a diagnosis of epilepsy. The data of the study was grouped into 4 sub-dimensions. A face-to-face interview method was conducted by the researcher using "The Child and Parent Introduction Form" and "The Pediatric Epilepsy Medication Self-management Questionnaire". Written informed consent was obtained from Akdeniz University Clinical Research Ethics Committee and the parents.

Results: Scoring received from 10 specialists was assessed to evaluate the scales scope validation and it was determined that there was agreement between the specialists (content validity index=0.89). The Kaiser-Meyer-Olkin value of 0.656 and Barlett's test value of ( $d f=210$, $p=0.000$ ) indicated that the data were sufficient in amount and suitable for factor analysis. As a result of exploratory factor analysis, 6 items for which factor loads were below 0.40 were removed from the scale. The factor structure of the newly formed 4 sub-dimension scale was tested by confirmatory factor analysis and the structure was confirmed $\left(\chi^{2} / \mathrm{df}=2.372\right.$, root mean square error of approximation $=0.079$, comparative fit index $=0.901$, goodness of fit index $=0.927$ and adjusted goodness of fit index $=0.851$ ). The Cronbach Alpha coefficient was found to be 0.71 for the total score of the scale. The sub-dimension correlations of the scale were determined to vary between 0.829-0.690. The testing correlations for test-retest were found to be significant $(p<0.001)$ and high $(0.91)$.
\end{abstract}

Conclusion: The Turkish adapted version of "The Pediatric Epilepsy Medication Self-management Questionnaire" was determined to band reliably for Turkish society.

Keywords: Epilepsy, self-management, psycholinguistics and psychometrics measurements, pediatric nursing

\section{Introduction}

Epilepsy is the most common disorder in childhood for which significant negative impacts are known in terms of the development of the child and the child's life quality
$(1,2)$. Although different statistics showing the prevalence of epilepsy are available, $4-10 \%$ of children worldwide experience a sort of attack before 20 -years-old and $1 \%$ of them are diagnosed with epilepsy (3). 
The self-management of epilepsy involves the participation of the child and their parents in daily activities, controlling symptoms generated by the illness and epilepsy itself, mitigation of the generated symptoms' effects on the quality of life and health status, regular utilization of medicines, development of a healthy lifestyle (sleep hygiene), participation in healthcare professionals' team decisions and coping with epilepsy (2,4-8). Based on current theories of self-management; parents whose children are diagnosed with epilepsy do not have the necessary knowledge about the self-management of epilepsy $(9,10)$, encounter difficulties in accessing healthcare professionals when they want to receive information (11), and so are not able to cope with epilepsy. Consequently, they experience a decrease in the quality of life, along with high levels of depression, anxiety and stress (12-14).

\section{Epilepsy and Cultural Characteristics}

Cultural characteristics play an important role towards the parents' self-management abilities of chronic diseases (3). Seizures cannot be controlled with antiepileptic drugs, especially resistant epilepsies, during childhood and parents use different traditional methods depending on their cultural beliefs $(15,16)$. When the literature is examined, it is seen that the knowledge and attitude of a society towards epilepsy differ with respect to cultural backgrounds (1722). Religious and socio-cultural beliefs affect the care and treatment processes of individuals diagnosed with epilepsy. In many developing societies, it is believed that epilepsy arises from witchcraft or possession by evil spirits. For this reason, it is not unheard of for treatment using healing herbs suggested by traditional doctors, priests, and religious leaders to be applied. Such practices can lead to a worsening of the symptoms and the development of complications $(23,24)$.

Keeping seizures under control by ensuring regular and recommended dosages of antiepileptic drugs and therefore improving the quality of life of the patient are key elements of the self-management of epilepsy. However, approximately $60 \%$ of children who receive antiepileptic treatment do not take their drugs as prescribed due to a lack of information about epilepsy and culture-specific reasons $(23,24)$. Providing accurate, up-to-date information about epilepsy to the individual diagnosed with epilepsy and their parents, and the development of self-management skills for both sides are among the most important components of the family-centred healthcare philosophy (25).

The care and maintenance process of individuals diagnosed with epilepsy and their parents is carried out under the guidance of a nurse and therefore nurses have a central role in the self-management of epilepsy (7). Therefore, it is very important for nurses to be knowledgeable about the different culture-specific practices used by parents in epilepsy self-management. When the literature is reviewed, only one scale is used to evaluate the self-management skills of the parents of children diagnosed with epilepsy in the international platform (5). In our country, there was no scale which could be used to determine the selfmanagement of the parents of children diagnosed with epilepsy and a such scale is a prerequisite for research in this area. The aim of this study is the Turkish adaptation of "The Paediatric Epilepsy Medication Self-management Questionnaire (PEMSQ)", the determination of its validityreliability, the evaluation of its suitability for Turkish culture, and a comparison of the psycholinguistic and psychometric properties of the scale with respect to different cultures.

\section{Materials and Methods}

\section{Participants and Setting}

The parents of all children who applied to Akdeniz University Hospital Paediatric Neurology Outpatient Clinic between April 2015 and June 2016 were the population of the study which is described as a methodological type. The sample group consisted of 540 parents of children aged between 2 and 17 years who had been diagnosed with epilepsy and who had been receiving antiepileptic treatment for at least six months. The size of the sample was calculated by taking into consideration the number indicated in the literature in order to enable the implementation of factor analysis. Based on the literature, a sample size corresponding to 50 is indicated as very weak, 100 as weak, 200 as a medium, 300 as good and 1,000 as excellent $(26,27)$.

\section{Data Collection}

After obtaining the necessary permissions to conduct the research, the data were collected by the researcher using the Description Form of the Child and Parent and the PEMSQ by means of face-to-face interviews with the parents in a quiet interview room at the Akdeniz University Paediatric Neurology Clinic. In order to determine the comprehensibility of the PEMSQ, after its translation processes, pre-application was carried out with 10 parents. As no problems were encountered in the preliminary application, the data collection process of the study was initiated. 


\section{Data Collection Tools}

The Description Form of the Child and Parent: It consists of 21 questions prepared in conformity with the related literature comprising information regarding the child (age, gender, duration of illness, frequency of seizure, regular drug usage, side effects of the drugs), their parents (ages of the mother and the father, education level) and knowledge on epilepsy.

PEMSQ: It is a 5-point Likert-type scale consisting of 27 items and 4 sub-dimensions developed by Modi et al. (5) to evaluate the self-management level of the parents of children diagnosed with epilepsy. The score from the scale varies between 27-135 and it is interpreted that selfmanagement skill increases as the score increases (5).

\section{Statistical Analysis}

The data of the study were evaluated in the SPSS (SPSS Inc., Chicago, IL) 23.0 package program. Since the data showed normal distribution after the normality test, the impact of independent variables (age, gender, etc.) on dependent variables (PEMSQ) were tested by application of t-test in pairwise groups and the One-Way ANOVA test was applied in groups of three or more to test the significance of the difference. The percentage and mean values were used to determine the descriptive characteristics of the parents and children who participated in the study. Adaptation and translation processes of the scales proposed by the World Health Organization for language validity were followed in the Turkish adaptation study of the PEMSQ (28) (Table I).

Psycholinguistic and Psychometric Measurements: The scale was translated and re-translated by six independent translators who have knowledge of the

Table I. Psycholinguistic and psychometric measurements of PEMSQ-TR

\begin{tabular}{|c|c|}
\hline $\begin{array}{l}\text { Psycholinguistic } \\
\text { measurements }\end{array}$ & Psychometric measurement \\
\hline Language validity & Validity methods \\
\hline \multirow{3}{*}{$\begin{array}{l}\text { Advanced translation } \\
\text { Expert panel } \\
\text { Re-translation } \\
\text { Pre-test } \\
\text { Final draft of the scale } \\
\text { Documentation }\end{array}$} & $\begin{array}{l}\text { Content validity (content validity } \\
\text { index) } \\
\text { Structural validity [exploratory } \\
\text { factor analysis (Kaiser-Meyer-Olkin, } \\
\text { Barlett test, confirmatory factor } \\
\text { analysis] }\end{array}$ \\
\hline & Reliability methods \\
\hline & $\begin{array}{l}\text { Internal consistency reliability } \\
\text { coefficient (Cronbach Alpha) } \\
\text { Test-retest method }\end{array}$ \\
\hline $\begin{array}{l}\text { PEMSQ-TR: Turkish Pediatric } \\
\text { Questionnaire }\end{array}$ & Epilepsy Medication Self-Management \\
\hline
\end{tabular}

language, who are familiar with the culture, who are experts in scale studies, and who know how the scales are used and interpreted based on the methodological part of the research report. Four translators translated the original scale into the target language, and the two translators retranslated the scale in the target language back to the original language.

The translations from the translators were reviewed by the researchers and, to obtain an expert opinion, the translations were sent to 10 faculty members in total; two from the child neurology field and eight from the field of paediatric nursing. Experts scored each item of the scale from 1 to 4 (1 point=not appropriate, 2 points=must be optimized, 3 points=appropriate but some small change required, 4 points=very appropriate) and these numerical values were evaluated by content validity index (CVI) analysis. As a result of the suggestions and evaluations from the experts, the final version of PEMSQ was created by the researchers. In order to determine the scale's comprehensibility, before the study, 10 parents completed the questionnaire in the outpatient clinic and no problems relating to comprehension were encountered.

In order to test the constructive validity of the scale, exploratory factor analysis (EFA) [Kaiser-Meyer-Olkin (KMO), Barlett's test] and confirmatory factor analysis were used (Table I). LISREL v8.8 (LISREL v8.8, Scientific Software International, Inc., Lincolnwood, IL) package program was used for confirmatory factor analysis (CFA). The timeinvariance test was evaluated by the test-retest method and the scale was reapplied to 50 parents at two-week intervals. The Cronbach alpha coefficient, which is commonly used in Likert type scales, was calculated to determine the internal consistency of the scale.

\section{Ethical Considerations}

In order to use the pre-research scale, permission was obtained from the author who originally developed the scale by e-mail. Ethical approval of the study was obtained from Akdeniz University Clinical Research Ethics Committee. Written informed consent was obtained from the parents who volunteered to participate in the study.

\section{Results}

\section{Sample Characteristics}

The majority of the children who participated in the study were in the 7-12-year age group, the mean age was $10.33( \pm 4.02)$ years and $52.2 \%$ of them were males. When their epilepsy and treatment characteristics were examined, 
it was determined that $44.4 \%$ of the children had been diagnosed with epilepsy more than 49 months prior, $50.3 \%$ had one or more seizures per year, $61.5 \%$ of them had forgotten their drug intake, $73.7 \%$ of them encountered side effects of the drugs and $86.9 \%$ came for check-ups on a regular basis. When the characteristics of the parents were examined, $49.1 \%$ of their mothers were between the ages of $31-40$ and $65.7 \%$ of them were primary school graduates. $48.5 \%$ of the fathers were aged 41 or over and $65.2 \%$ were primary school graduates. The mean age and gender of the children, the level of knowledge of their parents about epilepsy, the maternal age and the level of education of the parents were found to be related to the self-management of paediatric epilepsy on part of the parents (Table II).

Table II. Effect of demographic and disease related characteristics of participants on PEMSQ-TR Scores $(n=540)$

\begin{tabular}{|c|c|c|c|c|c|}
\hline Parameters & $\mathbf{n}$ & $\%$ & Mean (SD) & Statistic & p-value \\
\hline \multicolumn{6}{|c|}{ Characteristics of child } \\
\hline \multicolumn{6}{|l|}{ Age } \\
\hline $0-6$ & 116 & 21.5 & $2.98(0.27)$ & \multirow{3}{*}{$0.027^{a}$} & \multirow{3}{*}{$0.005^{*}$} \\
\hline 7-12 & 235 & 43.5 & $3.01(0.29)$ & & \\
\hline 13-18 & 189 & 35.0 & $3.32(0.31)$ & & \\
\hline \multicolumn{6}{|l|}{ Gender } \\
\hline Female & 258 & 47.8 & $3.25(0.30)$ & \multirow[b]{2}{*}{$0.414^{b}$} & \multirow[b]{2}{*}{$0.002^{*}$} \\
\hline Male & 282 & 52.2 & $3.01(0.38)$ & & \\
\hline
\end{tabular}

Features of epilepsy disease

How long has your child had epilepsy?

\begin{tabular}{|c|c|c|c|c|c|}
\hline 6-12 months & 81 & 15.0 & $3.18(0.39)$ & \multirow{5}{*}{$1.052^{c}$} & \multirow{5}{*}{0.380} \\
\hline 13-24 months & 98 & 18.1 & $3.25(0.34)$ & & \\
\hline 25-36 months & 69 & 12.8 & $3.24(0.31)$ & & \\
\hline $37-48$ months & 52 & 9.6 & $3.23(0.36)$ & & \\
\hline 49 months or over & 240 & 44.4 & $3.27(0.34)$ & & \\
\hline
\end{tabular}

Seizure frequency

Per week $\geq 1$

Per month $\geq 1$

Per year $\geq 1$

Per year $\leq 1$

\section{7}

\section{6}

\section{$(3.18(0.22)$}

\begin{tabular}{|l|l|}
91 \\
\hline 271 \\
61 \\
\hline
\end{tabular}

\section{Have you ever forgotten to give your child antiepileptic medicine?}

\begin{tabular}{|l|l|}
\hline Have you ever forgotten to give your child antiepileptic medicine? \\
\hline Yes & 332 \\
\hline No & 208 \\
\hline Are her any
\end{tabular}

\section{Are there any side effects of antiepileptic drugs?}

\begin{tabular}{l} 
Yes \\
\hline No \\
\hline
\end{tabular}

\begin{tabular}{|l|l|}
\hline 142 & 26.3 \\
\hline 398 & 73.7
\end{tabular}

\begin{tabular}{|l|l|}
\hline 61.5 & $3.23(0.35)$ \\
\hline 38.5 & $3.27(0.34)$ \\
\hline
\end{tabular}

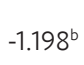

Are you attending check-ups regularly?

\begin{tabular}{|l|l|}
\hline Yes & 469 \\
\hline No & 71 \\
\hline
\end{tabular}

\begin{tabular}{|l|l|}
\hline 86.9 & $3.25(0.35)$ \\
\hline 13.1 & $3.19(0.33)$ \\
\hline
\end{tabular}

Have you been trained in epilepsy?

\begin{tabular}{|l}
\hline Yes \\
\hline No \\
\hline Somewhat \\
\hline
\end{tabular}

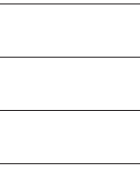

\begin{tabular}{|l|l|}
\hline 113 & 20 \\
\hline 372 & 68 \\
\hline 55 & 10 \\
\hline
\end{tabular}

\begin{tabular}{|l|l|l|l|}
\hline 20.9 & $3.40(0.32)$ & & \\
\cline { 1 - 2 } 68.9 & $3.20(0.35)$ & \multirow{2}{*}{$15,154^{c}$} & $0.000^{*}$ \\
\cline { 1 - 2 } 10.2 & $3.25(0.29)$ & & \\
\hline
\end{tabular}


Table II. contiuned

\begin{tabular}{|c|c|c|c|c|c|}
\hline \multicolumn{6}{|c|}{ The features of the mother } \\
\hline \multicolumn{6}{|l|}{ Age } \\
\hline $20-30$ & 129 & 23.9 & $3.19(0.25)$ & \multirow{3}{*}{$8,008^{c}$} & \multirow{3}{*}{$0.001^{*}$} \\
\hline $31-40$ & 265 & 49.1 & $3.47(0.30)$ & & \\
\hline 41 years or older & 146 & 27.0 & $3.22(0.35)$ & & \\
\hline \multicolumn{6}{|l|}{ Educational level } \\
\hline Literate & 53 & 9.8 & $2.73(0.30)$ & \multirow{4}{*}{$6,608^{c}$} & \multirow{4}{*}{$0.000^{*}$} \\
\hline Primary education & 355 & 65.7 & $3.23(0.33)$ & & \\
\hline High school & 115 & 21.3 & $3.34(0.35)$ & & \\
\hline University & 17 & 3.2 & $3.98(0.30)$ & & \\
\hline \multicolumn{6}{|c|}{ The features of the father } \\
\hline \multicolumn{6}{|l|}{ Age } \\
\hline $20-30$ & 51 & 9.4 & $3.24(0.27)$ & \multirow{3}{*}{$1.186^{c}$} & \multirow{3}{*}{0.751} \\
\hline $31-40$ & 227 & 42.1 & $3.03(0.31)$ & & \\
\hline 41 years or older & 266 & 48.5 & $3.18(0.34)$ & & \\
\hline \multicolumn{6}{|l|}{ Educational level } \\
\hline Literate & 22 & 4.1 & $2.96(0.28)$ & \multirow{4}{*}{$8,640^{c}$} & \multirow{4}{*}{$0.000^{*}$} \\
\hline Primary education & 352 & 65.2 & $3.19(0.32)$ & & \\
\hline High school & 133 & 24.6 & $3.31(0.40)$ & & \\
\hline University & 33 & 6.1 & $3.70(0.29)$ & & \\
\hline
\end{tabular}

\section{Validity Methods}

\section{CVI}

In assessing the scope validity of the PEMSQ, scoring findings from 10 experts were evaluated and CVI of 0.86 was determined.

\section{Structural Validity}

EFA and CFA were used to test the structural/constructive validity of the scale. In order to perform factor analysis, the suitability of the data set was firstly evaluated by KMO and Barlett's test. $\mathrm{KMO}=0.656$ and Barlett's test was found to be 4,691.86 ( $d f=210, p=0.000)$. As a result of the $E F A$, it was determined that the factor loads of items 5, 10, 15, 19, 21 and 27 of the scale consisting totally of 27 items (PEMSQ) were below 0.4 and these items were excluded from the scale. After the analysis of the remaining 21 items, the four-factor structure was formed and this structure explained 53,423\% of the total variance. The sub-dimensions of the newly formed structure are titled "information about epilepsy and treatment (F1)", "adaptation to treatment and clinical appointments (F2)", "treatment-related obstacles (F3)" and "treatment and social life (F4)" (Table III). In order to determine whether the 21-item structure of the 4 subdimensions of the scale was verified, CFA was applied.

The path diagram is shown in Figure 1. The fit indices were determined as follows: $\chi^{2} / \mathrm{df}=2.372$, root mean square error of approximation (RMSEA) $=0.079$, comparative fit index $(\mathrm{CFI})=0.901$, goodness of fit index $(\mathrm{GFI})=0.927$ and adjusted goodness of fit index $(\mathrm{AGFI})=0.851$. When the coefficients of the relationship between the observed variables and the factors showing the factor structure of this scale were examined, it was concluded that all coefficients were at sufficient levels. Considering the compliance statistics calculated by CFA, it has been decided that the previously determined structure of the scale conforms to the acceptable level with the collected data (Table IV). 
Table III. Factor structure, loading and variance of PEMSQ-TR explained

\section{Items}

Information about epilepsy and treatment

(1) Doctors/Nurses fully explained the diagnosis of seizures/epilepsy

(2) I know which side effects I should follow in my child while the treatment is in progress

(3) I know whom to contact when I encounter any problems

(4) I believe that I can manage in cooperation with the health team when any side effects occur

(5) I know the risks that my child may face if the drug is discontinued before a 2-year period without seizure

(6) The medical team listens to my concerns about my child

(7) It is easy for me to reach my child's healthcare providers and they will respond to my questions immediately

(8) I am not having trouble bringing my child to appointments

(9) Drug treatment is necessary for my child's disease

(10) Medications given for treatment will control the seizures of my child

\begin{tabular}{|l|l|l|l|}
\hline \multicolumn{4}{|c|}{ Factor } \\
\hline (F1) & (F2) & (F3) & (F4) \\
\hline
\end{tabular}

\section{Adaptation to treatment and clinical appointments}

(11) I check that my child has taken his medicine as prescribed

(12) I usually follow the mentioned medical advice and treatment plans for myself and my child

(13) I think it is important for my child to take his treatment as described

(14) All members of the family comply with my child's treatment plan

(15) I think it is important to provide the medication my child should take daily

\begin{tabular}{|l|l|l|l|}
\hline 0.580 & & & \\
\hline 0.844 & & & \\
\hline 0.631 & & & \\
\hline 0.588 & & & \\
\hline 0.749 & & & \\
\hline 0.600 & & & \\
\hline 0.612 & & & \\
\hline 0.593 & & & \\
\hline 0.515 & & & \\
\hline 0.531 & & & \\
\hline
\end{tabular}

\section{Treatment-related obstacles}

(16) My child does not like the taste of the drugs

(17) My child has difficulty in taking the medicine

(18) My child refuses to take his medicine

(19) There are times when our medicine is depleted

\begin{tabular}{|l|l|l|l|}
\hline & 0.598 & & \\
\hline & 0.693 & & \\
\hline & 0.834 & & \\
\hline & 0.581 & & \\
& 0.558 & & \\
& & 0.750 & \\
\hline & & 0.822 & \\
\hline & & 0.790 & \\
\hline & & 0.449 & \\
\hline
\end{tabular}

\section{Treatment and social life}

(20) My child is ashamed of taking their medicine in front of others (friends, family, etc.)

(21) My child has some activities (sports, school activities, etc.) that may interfere with drug intake

Cumulative variance

Variance explained (\%)

Cumulative explained variance (\%)

\section{Reliability Methods}

\section{Internal Consistency Reliability Coefficient}

As a result of the factor analysis, the total scale and Cronbach Alpha values of each dimension were calculated. The overall Cronbach Alpha coefficient of the scale was 0.71 and the sub-dimensions were 0.829 (first sub-dimension: F1), 0.732 (second sub-dimension: F2), 0.726 (third subdimension: F3), and 0.692 (fourth sub-dimension: F4).

\section{Test-Retest Method}

Evaluation of invariance in the scale was carried out by the test-retest method and the scale was reapplied with 50 parents at two week-long intervals. The scores between the two applications were evaluated via the Pearson productmoment correlation test and the correlation coefficient was determined to be $r=0.91$. 


\section{Discussion}

Epilepsy is the most common $(0.5 \%$ to $1 \%)$ chronic neurological disease in childhood, and there are many problems related to antiepileptic treatment due to a lack of knowledge about the disease and also culture-specific reasons (29). When the literature is examined; it is seen that approximately $60 \%$ of children receiving antiepileptic therapy are not using their drugs as prescribed and they are not provided with regular polyclinic check-ups. Additionally, some of the parents whose children have resistant epilepsy think that antiepileptic treatment is ineffective and they use different traditional methods depending on their cultural beliefs $(16,23,24,30)$. Taking all the above-mentioned reasons into consideration, it is very important to promote selfmanagement for the effective management and treatment of epilepsy.

Self-management of chronic diseases such as epilepsy involves the control of the symptoms of the disease, compliance with the planned treatment regimen, interaction with health care professionals, making lifestyle changes to improve health, and coping with the disease based on feelings with a problem-focused approach

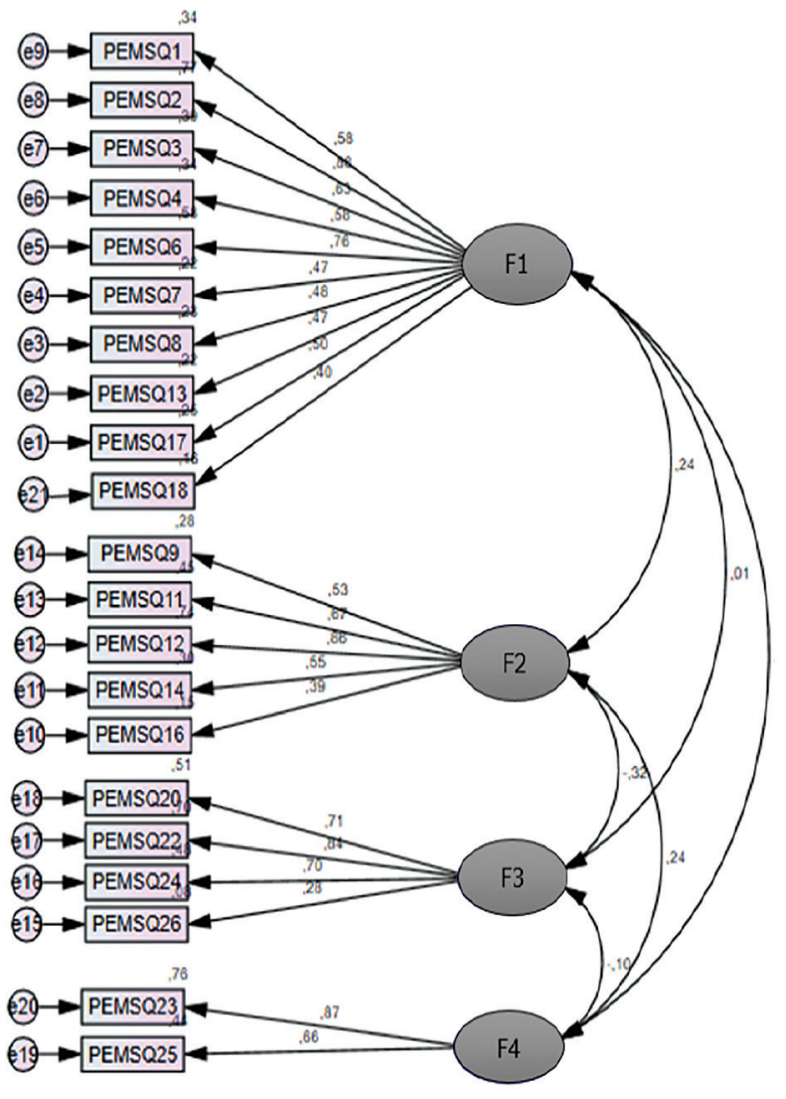

Figure 1. Path diagrams

PEMSQ: Pediatric Epilepsy Medication Self-management Questionnaire
$(2,5,6,31)$. When the literature is examined, it is seen that the PEMSQ developed by Modi et al. (5) in English is the only measurement tool to evaluate the self-management of the parents of children diagnosed with epilepsy. In addition, the adaptation of the scale into different languages or cultures was not observed. For this reason, in our study, we aimed to determine the adaptation, validity and reliability of the Turkish PEMSQ (PEMSQ-TR) and the findings are discussed in three sections.

\section{Sample Characteristics}

In our study, it was determined that the results of the "PEMSQ" were related to the average age of the children, maternal age, the knowledge of the participants about the disease and the level of education of the parents. It was determined that success in the self-management of epilepsy positively correlated with the age of the children, the maternal age, the knowledge level of the parents regarding the illness and the education backgrounds of the parents $(p<0.05)$ (Table II). When the literature is examined, it is stated that there are similar findings with our study results, and as the level of knowledge about epilepsy increases, the levels of self-management and coping with the disease are specified to be better $(6,32)$.

\section{Validity Methods}

\section{CVI}

CVI technique was used as a criterion for evaluating both the language and culture equivalence and content validity of the scale with numerical values and for evaluating the expert opinions. In this technique, the scale has to be 0.80 or above to indicate that the scale has scope validity (33). PEMSQ-TR's scoring scores from 10 experts were evaluated and it was determined that $C V I=0.89$. This value shows that the scope validity of the scale is at a good level.

\section{Structural/Construct Validity}

Before the construct validity is tested, the suitability of the data set for factor analysis is determined. For this purpose, the Barlett's test is applied to determine whether or not KMO and variables are correlated with each other. A KMO value of less than 0.50 indicates that the sample size is not sufficient for validity analysis (34). One of the commonly used methods to test the construct validity in scale studies is factor analysis. Factor analysis tests the integrity of the scale and helps to eliminate unrelated variables. Items that are highly related among themselves form factors. Factor analysis is done by two methods which are exploratory and confirmatory factor analysis $(33,35)$. 


\begin{tabular}{|c|c|c|c|}
\hline Index of compliance & Excellent compliance limit & Acceptable compliance limit & PEMSQ-TR \\
\hline RMSEA & $0<$ RMSEA $<0.05$ & $0.05<$ RMSEA $<0.10$ & 0.079 \\
\hline GFI & $0.95<\mathrm{GFI}<1$ & $0.90<\mathrm{GFI}<0.95$ & 0.927 \\
\hline AGFI & $0.90<$ AGFI $<1$ & $0.85<$ AGFI $<0.90$ & 0.851 \\
\hline $\mathrm{CFI}$ & $0.95<\mathrm{CFI}<1$ & $0.90<C F \mid<0.95$ & 0.901 \\
\hline$\chi^{2} / \mathrm{df}$ & $\chi 2 / d f<3$ & $3<\chi 2 / d f<5$ & 2.372 \\
\hline
\end{tabular}

In our study, it was determined that the sample size was sufficient $(\mathrm{KMO}=0.656)$ and the data set was suitable for factor analysis ( $d f=210, p=0.000$ ). According to Harrington (36), factor loads should not be less than 0.30. Factor loads of 0.71 or above are deemed to be excellent, 0.63 very good, 0.55 good, 0.45 acceptable and 0.32 or less is deemed to be weak. In our study, 6 items $(5,10,15,19,21$ and 27 items) with a factor load below 0.4 were excluded from the original scale. After the removal of these items, a 4-dimensional structure for 21 items was formed which explained 53,423\% of the total variance. Henson and Roberts (37) stated that the variance explained in scale studies should be $52 \%$ or more. In our study, it was seen that the explained variance is above the stated value and the newly formed structure was approved.

In order to determine whether the 21-item structure of the 4 sub-dimensions of the scale was verified, DFA was applied. For the construct validity of a scale, the fit indices made in the confirmatory factor analysis should be at the desired level. For the model to be acceptable, the chi-square value should be 2 or less indicating that the model is a good model, and if it is 5 or less, it indicates that the model has an acceptable goodness of fit $(33,35)$. If the RMSEA value is less than or equal to 0.80 , it indicates that the compatibility is good, if it is equal to or less than 0.10 , it indicates that the compatibility is low. When the CFI and GFI values are equal to or greater than 0.90 , it indicates that there is compatibility (33). In our study, the fit index of the scale consisting of 4 sub-dimensions of 21 items was found to be at a good level and the new scale structure was approved.

\section{Reliability Methods}

\section{Internal consistency (Cronbach Alpha)}

It should be determined that each item of the scale measures the same attitude within itself. The most appropriate method for this is the calculation of the Cronbach Alpha reliability coefficient. A Cronbach Alpha coefficient between $0.00 \leq \alpha \leq 0.040$ indicates that the scale is not reliable, between $0.40 \leq \alpha \leq 0.60$ indicates that reliability of the scale is low, between $0.60 \leq \alpha \leq 0.80$ indicates that reliability of the scale is satisfactory and between $0.80 \leq \alpha \leq 1.00$ indicates that reliability of the scale is high (38). In parallel with the literature, it was concluded that the internal consistency coefficient of the scale was satisfactory and the scale was reliable.

\section{Test-retest method}

When the same measurement tool is applied to the same individuals at different times, the similarity of the responses given by the individuals to the measurement tool indicates the invariance of the measurement tool. The correlation coefficient ( $r$-value) between the first and second application scores of this invariance is calculated by the Pearson Product Moment Correlation test. The closer this value is to 1 , the better the reliability is, but the value should be at least 0.70 and a value above 0.80 is preferred (39). In our study, it was found that the value of " $r$ " was very close to $1(r=0.91)$. It was concluded that the scale was consistent with respect to time.

\section{Conclusion}

In our study, the psycholinguistic and psychometric measurements of the PEMSQ-TR were evaluated. As a result of the analyses, it was determined that PEMSQ-TR is a valid and reliable measurement tool for the evaluation of the disease self-management of those parents with epileptic children.

Ethics

Ethics Committee Approval: Ethical approval for this study was obtained by Akdeniz University Faculty of Medicine Clinical Research Ethics Committee (approval no: 177, date: 15.04.2015). 
Informed Consent: Written informed consent was obtained from the parents who volunteered to participate in the study.

Peer-review: Externally peer-reviewed.

\section{Authorship Contributions}

Concept: Ş.T., A.I.D., Design: Ş.T., Data Collection: Ş.T., Analysis or interpretation: Ş.T., Writing: Ş.T., A.I.D.

Conflict of Interest: The authors declared no conflict of interest.

Financial Disclosure: The authors declared that this study received no financial support.

\section{References}

1. Kariuki SM, Newton CR, Prince MJ, Das-Munshi J. The association between childhood seizures and later childhood emotional and behavioral problems: findings from a nationally representative birth cohort. Psychosom Med 2016; 78:620.

2. Guilfoyle SM, Wagner LL, Modi AC, et al. Pediatric epilepsy and behavioral health: The state of the literature and directions for evidence-based interprofessional care, training, and research Clin Pract Pediatr Psychol 2017; 5:79-90.

3. Duffy LV. Parental coping and childhood epilepsy: the need for future research. I Neurosci Nurs 2011; 43:29-35.

4. Ryan P, Sawin KJ. The individual and family self-management theory: Background and perspectives on context, process, and outcomes. Nurs Outlook 2009; 57:217-25.

5. Modi AC, Monahan S, Daniels D, Glauser TA. Development and validation of the pediatric epilepsy medication selfmanagement questionnaire. Epilepsy Behav 2010; 18:94-9.

6. Modi AC, Wu YP, Guilfoyle SM, Glauser TA. Uninformed clinical decisions resulting from lack of adherence assessment in children with new-onset epilepsy. Epilepsy Behav 2012; 25:481-4.

7. Lewis SA, Noyes 1, Hastings RP. Systematic review of epilepsy self-management interventions integrated with a synthesis of children and young people's views and experiences. I Adv Nurs 2015; 71:478-97.

8. Fleeman N, Bradley PM. Care delivery and self-management strategies for children with epilepsy. Cochrane Database of Syst Rev 2018; CD006245. doi: 10.1002/14651858.CD006245.pub4

9. Carbone L, Zebrack B, Plegue M, Joshi S, Shellhaas R. Treatment adherence among adolescents with epilepsy: what really matters? Epilepsy Behav 2013; 27:59-63.

10. Tekle-Haimanot R, Pierre-Marie P, Daniel G, Worku DK, Belay HD, Gebrewold MA. Impact of an educational comic book on epilepsy-related knowledge, awareness, and attitudes among school children in Ethiopia. Epilepsy Behav 2016; 61:218-23.

11. McNelis AM, Buelow J, Myers J, Johnson EA. Concerns and needs of children with epilepsy and their parents. Clin Nurse Spec 2007; 21:195-202.

12. Wagner JL, Smith G, Ferguson P, van Bakergem K, Hrisko $S$. Pilot study of an integrated cognitive-behavioral and self-management intervention for youth with epilepsy and caregivers: Coping Openly and Personally with Epilepsy (COPE). Epilepsy Behav 2010; 18:280-85.
13. Kerr C, Nixon A, Angalakuditi M. The impact of epilepsy on children and adult patients' lives: development of a conceptual model from qualitative literature. Seizure 2011; 20:764-74.

14. Puka K, Tavares TP, Anderson KK, Ferro MA, Speechley KN. A systematic review of quality of life in parents of children with epilepsy. Epilepsy Behav 2018; 82:38-45.

15. Hockenberry MJ, Wilson D, Rodgers CC. Wong's Essentials of Pediatric Nursing-E-Book. Elsevier Health Sciences, 2016.

16. Tutar Güven S, İşler Dalgıç A. Antiepileptic medications used for children with epilepsy and the importance of nursing approach in medication administration. International Refereed Journal of Nursing Researches 2017; 9:188-207.

17. Gazibara T, Nikolovski J, Lakic A, Pekmezovic T, Kisic-Tepavcevic D. Parental knowledge, attitudes, and behaviors towards children with epilepsy in Belgrade (Serbia). Epilepsy Behav 2014; 41:210-6.

18. Pupillo E, Vitelli E, Messina P, Beghi E. Knowledge and attitudes towards epilepsy in Zambia: a questionnaire survey. Epilepsy Behav 2014; 34:42-6.

19. Maiga $\mathrm{Y}$, Albakaye $\mathrm{M}$, Diallo LL, et al. Current beliefs and attitudes regarding epilepsy in Mali. Epilepsy Behav 2014; 33:11521.

20. Ak PD, Atakli D, Yuksel B, Guveli BT, Sari H. Stigmatization and social impacts of epilepsy in Turkey. Epilepsy Behav 2015; 50:50-4.

21. Aydemir N, Kaya B, Yıldız G, Öztura I, Baklan B. Determinants of felt stigma in epilepsy. Epilepsy Behav 2016; 58:76-80.

22. Doganavsargil-Baysal O, Cinemre B, Senol Y, Barcin E, Gokmen Z. Epilepsy and stigmatization in Turkey. Epilepsy Behav 2017; 73:100-5.

23. Kabir M, Iliyasu Z, Abubakar IS, Kabir ZS, Farinyaro AU. Knowledge, attitude and beliefs about epilepsy among adults in a northern Nigerian urban community. Ann Afr Med 2005; 4:107-12.

24. Modi AC, Guilfoyle SM, Mann KA, Rausch JR. A pilot randomized controlled clinical trial to improve antiepileptic drug adherence in young children with epilepsy. Epilepsia 2016; 57:e69-75. doi: 10.1111/epi.13289.

25. England MJ, Liverman CT, Schultz AM, Strawbridge LM, Institute of Medicine (US) Committee on the Public Health Dimensions of the Epilepsies. Educating People with Epilepsy and Their Families, Washington (DC): National Academies Press (US), 2012.

26. Çokluk Ö, Şekercioğlu G, Büyüköztürk Ş. Multivariate statistics for the social sciences: SPSS and LISREL applications. Ankara: Pegem Akademi, 2016.

27. International Test Commission (ITC). Guidelines for translating and adapting tests (Second Edition). Int J Test 2018; 18:101-34.

28. World Health Organisation (WHO). Process of translation and adaptation of instruments. Last Accessed Date: 29.01.2019. Available from: https://www.who.int/substance_abuse/ research tools/translation/en/

29. Aaberg KM, Gunnes N, Bakken II, et al. Incidence and prevalence of childhood epilepsy: a nationwide cohort study. Pediatrics 2017; 139:e20163908. doi: 10.1542/peds.2016-3908.

30. Tutar Güven \$̧, Işler A. Importance of seizure self-efficacy in children with epilepsy for disease management. Epilepsi 2014; 103:106. 
31. Unger WR, Buelow JM. Hybrid concept analysis of selfmanagement in adults newly diagnosed with epilepsy. Epilepsy Behav 2009; 14:89-95.

32. Paschal AM, Mitchell QP, Wilroy JD, Hawley SR, Mitchell JB. Parent health literacy and adherence-related outcomes in children with epilepsy. Epilepsy Behav 2016; 56:73-82.

33. Burns N, Grove SK. Understanding Nursing Research-eBook: Building an Evidence-Based Practice. Elsevier Health Sciences, 2010.

34. Thompson B. Exploratory and confirmatory factor analysis: Understanding concepts and applications. American Psychological Association, 2004.
35. Civelek ME. Essentials of structural equation modeling. Lincoln, Nepraska, Zea Books, 2018.

36. Harrington D. Confirmatory factor analysis. Oxford University Press, 2009

37. Henson RK, Roberts JK. Use of exploratory factor analysis in published research: Common errors and some comment on improved practice. Educ Psychol Meas 2006; 66:393-416.

38. Kılıç S. Cronbach's alpha reliability coefficient. I Mood Disord 2016; 6:47.

39. Polit DF, Beck CT. Essentials of nursing research: Appraising evidence for nursing practice. 7th ed. Philadelphia, Wolters Kluwer Health, Lippincott Williams \& Wilkins, 2010. 